\title{
Astrometric detection of faint companions - the Pluto/Charon case study
}

\author{
A. H. Andrei ${ }^{1,2}$, V. Antunes Filho ${ }^{2}$, R. Vieira Martins ${ }^{1}$, M. Assafin ${ }^{2}$, \\ D.N. da Silva Neto ${ }^{2,3}$ and J. I. B. Camargo ${ }^{2}$ \\ ${ }^{1}$ Observatório Nacional/MCT \\ R. Gal. Jos Cristino 77, RJ, Brasil \\ email: oat1@on.br \\ ${ }^{2}$ Observatório do Valongo/UFRJ, Brasil \\ ${ }^{3}$ Universidade Estadual da Zona Oeste/RJ, Brasil
}

\begin{abstract}
The resolution of pairs of objects closer than the scale of seeing, and of difference of magnitude larger than ten percent is unreliable by direct imaging. The resulting image FWHM differs from a true PSF by no more than four percent. Yet, the peak of the associated Gaussian is shifted to a larger proportion.

The main results are the description of the FWHM and peak location shifts as function of the seeing scale, the centers separation, and of the magnitudes difference. Analytically, the estimators of variation were the resulting Gaussian amplitude, mean value, and standard deviation. The later is shown to be the most reliable estimator.
\end{abstract}

Keywords. methods: data analysis, techniques: image processing, astrometry, planets and satellites: general, binaries: general

\section{Results}

Figure 1 presents, for the Pluto/Charon case, the composition of the profiles of the brighter and dimmer companions. It is seen that the resulting compound curve is well represented by a Gaussian which differs from a single object PSF. The analysis of the ensemble of PSFs in the field permits to indicate the one revealing the signature of a compound system.

Figure 2 presents the nearly helicoidal wandering of the photocentre of the compound image of the Pluto/Charon system (i.e., without intervention of the dynamical pulling), from the survey campaign at the $0.6 \mathrm{~m}$ B\&C telescope at the LNA, Itajubá, Brasil. On the right, the same effect for a single Charon's orbit.

Once correcting the observed image from the photocentre wandering effect, the true Pluto's observed positions can be compared with its ephemeris. Systematics trends are seen both in RA (left) and DEC (right), the former with amplitude about twice of the latter. The trends cannot be of observational nature, due to the time span (6 years). Instead they can be reconciled to RA and DEC ephemeris corrections for the Pluto's orbit, matching recent determinations (Sicardy et al., 2006). 


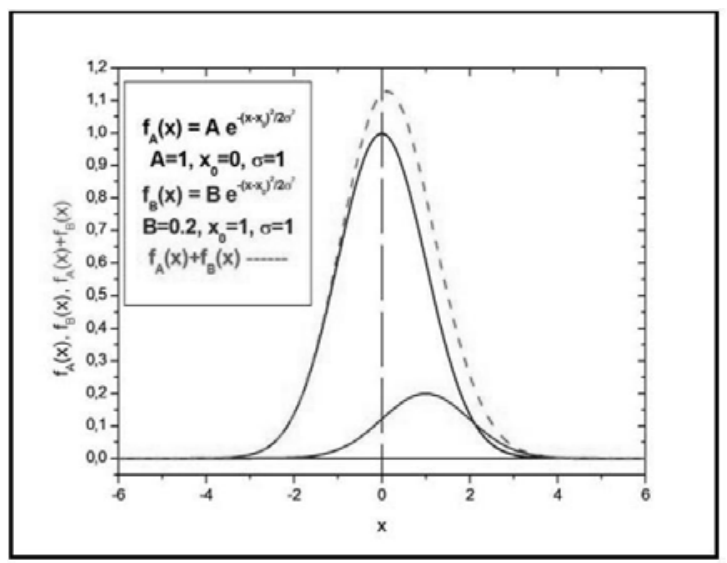

Figure 1. Gaussian composition (dashed curve) of the brighter (continuos line, taller curve) and dimmer companions (continuos line, shorter curve)

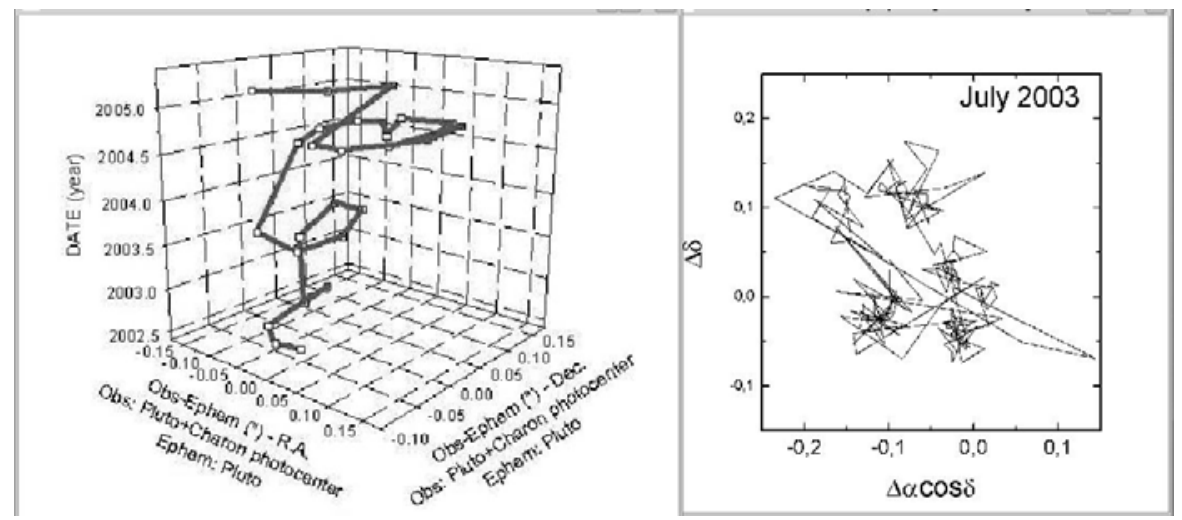

Figure 2. Charon's signature upon the astrometric variation of the compound photocentre of the Pluto/Charon system.

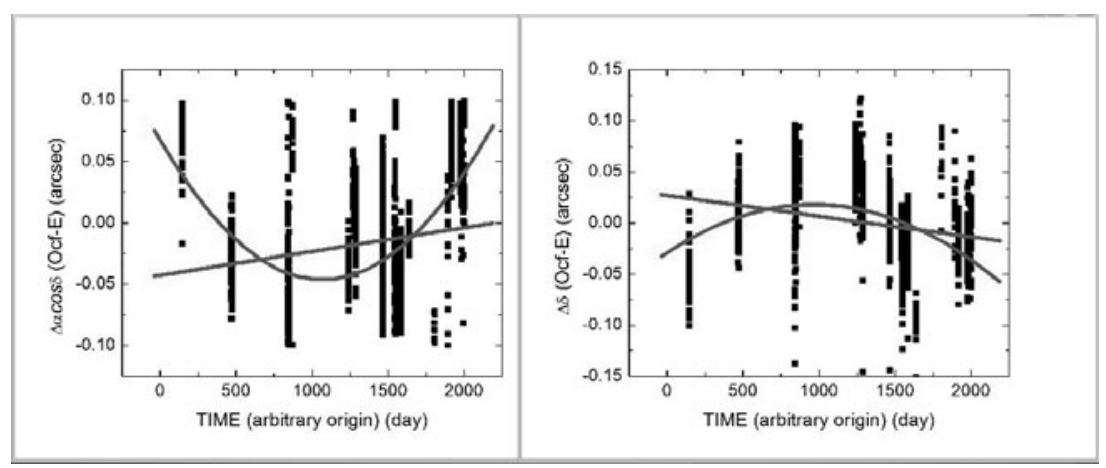

Figure 3. Linear and Second degree fits to series of Pluto observed positions, once corrected from the photocentre wandering, minus the ephemeris.

\section{References}

Sicardy, B., Bellucci, A., Gendron, E. et al. 2006, Nature 439, 52 